\title{
Le Parc National D'al Hoceima: Panorama Entre Terre Et Mer
}

\author{
Sarra El Hadri, \\ Laboratoire Botanique Appliquée, Faculté des \\ Sciences, Université Abdelmalek Essaâdi, Tétouan, Maroc \\ Anouar Jaoui, \\ Direction des Eaux et Forêts, Maroc \\ Hassan Bouziane, \\ Laboratoire Botanique Appliquée, Faculté des \\ Sciences, Université Abdelmalek Essaâdi, Tétouan, Maroc
}

Doi:10.19044/esj.2019.v15n27p311 URL:http://dx.doi.org/10.19044/esj.2019.v15n27p311

\section{Résumé}

Conserver, protéger et valoriser sont des piliers principaux de la création des Parcs Nationaux au Maroc. Citant le Parc National d'Al Hoceima caractérisant par des exclusivités parmi eux on cite ; la présence des espèces emblématiques tel que le balbuzard pêcheur et le thuya qui sont le symbole de logo de ce parc ; le seul au Maroc qui englobe une partie terrestre et l'autre partie marine et qui a une vue sur la mer méditerranée ce qui résume un panorama agréable entre terre et mer, avec un aspect montagneux composé des falaises et des grottes, qui constitue un habitat de phoque moine qui a disparu et certains espèces ornithologues comme le balbuzard pêcheur, des îlots et des rochers abritant plusieurs espèces des reptiles. Ce Parc est un endroit qui réponds aux besoins des amateurs pratiquant leurs loisirs et aussi c'est une destination pour les chercheurs qui participent à la gestion et à la préservation de la biodiversité de ce milieu ce qui impose l'existence des certains gites en assurant le confort et la détente des visiteurs dans ce milieu naturel afin de développer le tourisme durable avec la bonne pratique de la conservation de la nature.A partir de cet article on met l'accent sur l'avifaune qui est le balbuzard pêcheur, aussi sur l'espèce végétal concernant le thuya de Berbérie en matière de suivi et conservation sans oublié l'écotourisme qui joue un rôle principale dans le développement durable du Parc National D'al Hoceima en impliquant le principe de la proche participative.

Mot-clés : Parc, Al Hoceima, Biodiversité, Tourisme, Développement 


\title{
The National Park of Al Hoceima: Panorama Between Land and Sea
}

\author{
Sarra El Hadri, \\ Laboratoire Botanique Appliquée, Faculté des \\ Sciences, Université Abdelmalek Essaâdi, Tétouan, Maroc \\ Anouar Jaoui, \\ Direction des Eaux et Forêts, Maroc \\ Hassan Bouziane, \\ Laboratoire Botanique Appliquée, Faculté des \\ Sciences, Université Abdelmalek Essaâdi, Tétouan, Maroc
}

\begin{abstract}
Conserving, protecting and enhancing are the main pillars of the creation of National Parks in Morocco. Citing the Al Hoceima National Park characterizing by exclusivities among them one quotes; the presence of emblematic species such as the osprey and cedar, which are the logo symbol of this park, the only one in Morocco that includes a land part and the other marine part and has a view of the Mediterranean Sea, which summarizes a pleasant panorama between land and sea, with a mountainous aspect composed of cliffs and caves that constitute a habitat for monk seals that has disappeared and some bird species such as the osprey, islets and rocks housing several reptile species. This park is a place that meets the needs of land and marine enthusiasts practicing their leisure activities and is also a destination for researchers who participate in the management and preservation of the biodiversity of this environment, which requires the existence of certain cottages by ensuring the comfort and relaxation of visitors in this natural environment in order to develop sustainable tourism with good practice in nature conservation. From this article we focus on the avifauna which is the osprey, also on the plant species concerning the Berber cedar in terms of monitoring and conservation without forgetting the ecotourism which plays a main role in the sustainable development of Al Hoceima National Park by involving the principle of close participation.
\end{abstract}

Keywords: Park, Al Hoceima, Biodiversity, Tourism, Development 


\section{Introduction}

$\mathrm{Au}$ Maroc, les forêts occupent une place remarquable parmi les écosystèmes méditerranéens, dû aussi bien à leur haute biodiversité qu'aux services écologiques et environnementaux qu'ils offrent (Et-tobi et al., 2008). Ils se constituent principalement par deux tiers des espèces végétales et un tiers d'espèces animales. Son rôle reste d'autant plus important que c'est au sein des écosystèmes forestiers que s'élaborent les grands cycles, comme celui de l'eau (HCEFLCD).

Ces forêts, soient elles, urbaines ou périurbaines sont soumises à des pressions multiples : urbanisation croissante, fréquentation non organisée, convoitise foncière, ...ces dynamiques non maîtrisées compromettent l'équilibre environnemental et la pérennité des écosystèmes forestiers (HCEFLCD, 2010). Et pour faire face à ces problèmes environnementaux le Maroc a pris la décision de la création des aires protégées pour conserver, protéger et valoriser les ressources naturelles. Depuis 1942 jusqu'au 2006 le Maroc a créée neuf Parcs Nationaux qui représentent la biodiversité du pays. La création de ses Parcs nécessite l'intégration de la population locale au développement économique (activité humaine, valorisation, etc.) dans le sens de la gestion intégrale des ressources naturelle. Cette association de notion d'intégration de l'activité humaine à la création ou au développement d'un Parc National quel qu'il soit et où il se trouve ne veut pas dire destruction du milieu, mais seulement un développement adéquat et équilibré en rapport avec la consommation locale et régional (Najim, 2001). Donc le rôle principale du Parc n'est pas juste la protection des espèces mais il est aussi un objet pédagogique : il permet de répandre la connaissance et le respect de la nature en encourageant un éco-tourisme par des aménagements de parkings paysagers de sentiers botaniques ou encore l'organisation de manifestations touristiques.

\section{La zone d'étude :}

Situé au nord du Maroc, le long de sa côte méditerranéenne, à $150 \mathrm{~km}$ environ à l'est du détroit de Gibraltar, à proximité de la ville d'Al Hoceima $\left(4^{\circ} \mathrm{N}, 35^{\circ} 14^{\prime} \mathrm{N}\right)$. Il a été créé en octobre 2004 au sud de la mer d'Alboran sur une superficie de 48460 ha dont 19000 ha en zone marine, c'est la plus importante aire protégée de la côte méditerranéenne du Maroc et c'est l'unique Parc National au Maroc sur la façade méditerranéenne qui comporte une partie marine avec plusieurs îlots et rochers (Atlas du Parc National d'al Hoceima). Le Parc national d'Al Hoceima est une enclave naturaliste, culturelle et historique unique en son genre pour le Maroc (Brochure, Ministère des Eaux et Forêts). Il abrite une biodiversité importante après la Turquie au niveau de la méditerranée avec un taux d'endémisme de $20 \%$. 


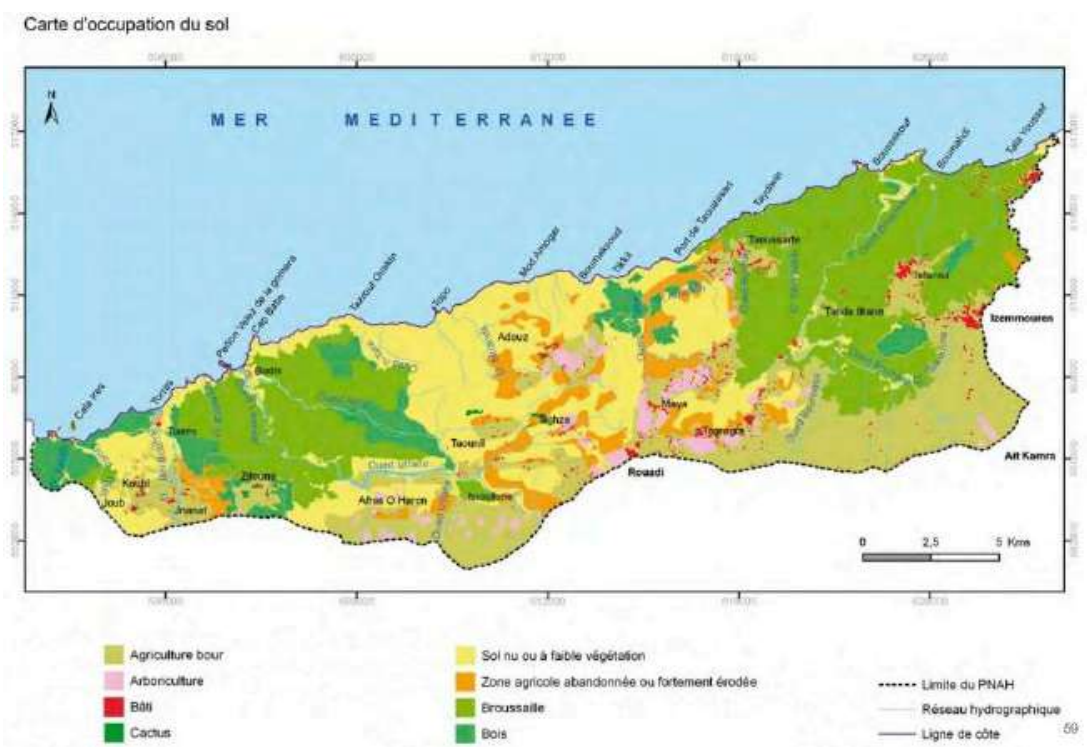

Figure 1: Carte d'occupation du sol du PNAH (source : Atlas du parc national d'al Hoceima)

Les objectifs du PNAH sont : la conservation d'échantillons représentatifs du patrimoine naturel de la façade méditerranéenne du Maroc, le maintien des équilibres naturels et des processus écologiques vitaux, la préservation de la diversité biologique et de la complémentarité des habitats naturels de l'ensemble du Parc, L'information, l'éducation et la sensibilisation de différents publics, la protection des paysages caractéristiques du Parc, la mise en place de conditions particulières pour un développement local et une amélioration des conditions de vie, par la réalisation de programmes de développement intégré et participatif (Tunesi et al., 2003).

\section{Une richesse climatique, géologique et biologique \\ - Climat :}

Le Parc National d'Al Hoceima se caractérise par un climat méditerranéen sec qui appartient à un étage bioclimatique méditerranéen aride supérieur à semi-aride.

Il montre un été chaud et sec de juin à novembre et un hiver frais et humide de décembre à avril. La moyenne des maxima du mois le plus chaud est de $28.5^{\circ} \mathrm{C}$ et la moyenne des minimums du mois le plus froid est de $10^{\circ} \mathrm{C}$ sur la côte et correspond au mois de janvier. 


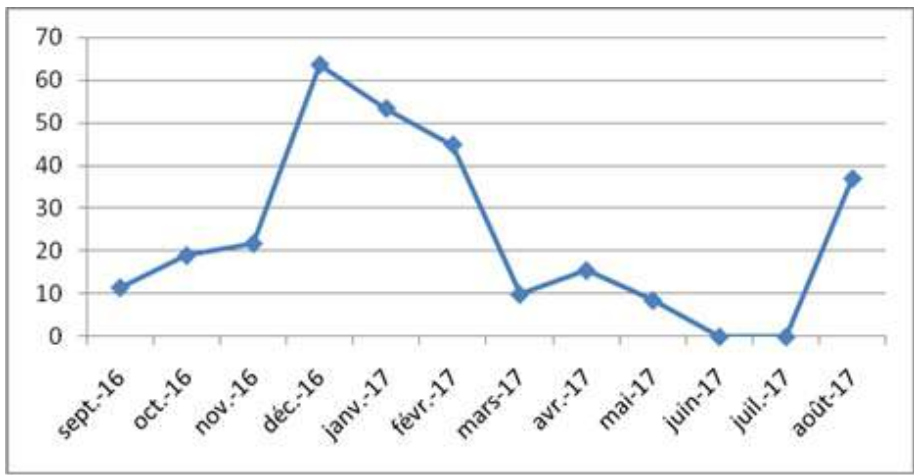

Graphe 1 : représente les précipitations de la commune Béni Boufrah de l'année 2016/2017(source : DPEF)

Les précipitations, souvent sous formes d'averses intenses et brutales sur les bassins versants à forte pente, accentuent l'érosion et la perte en sol, surtout dans les terrains sans ou à rare couvert végétal. D'après le graphe (graphe 1) on constate que les pluviométries se commencent en saison d'hiver (septembre-octobre-novembre) avec une moyenne de 17,43 $\mathrm{mm}$, les pluies se continue jusqu'ils arrivent à un maximum au mois de décembre avec $63,6 \mathrm{~mm}$, puis ils se diminuent jusqu'à sa disparition en saison d'été avec un total de $285,1 \mathrm{~mm} / \mathrm{an}$. En hiver et au printemps, les vents sont de secteur ouest à sudouest, avec parfois de courtes mais violentes tempêtes d'est. Durant la saison sèche, été et automne, le régime général est d'est à nord-est (le "chergui"), avec alternance journalière de brises de mer et de terre. Ce vent sec et chaud est parfois remplacé, pour de courtes durées, par un régime d'ouest. (Tunesi et al., 2003)

\section{- Géologie :}

Sur le plan géologique, le littoral, dans sa plus grande partie, montre une structure comparable à celle du Rif oriental avec, du sud au nord, des unités structurales allant des plus basses vers les plus élevées (le massif des Bokkoyas) (Atlas du parc national d'al Hoceima). Au carrefour d'un ensemble géologique (Rif Central) et de zones sédimentaires (hautes vallées du nordouest du Parc), la diversité géologique du massif se traduit notamment par un relief cloisonné et des sols de nature variée. Les influences climatiques multiples qui marquent cet espace s'expliquent en raison de la confluence des zones méditerranéenne, rifaine et continentale, et de l'existence d'un gradient altitudinal marqué à proximité de la côte. Ces facteurs physiques sont à l'origine d'une remarquable diversité des milieux naturels, qui permet la présence d'un grand nombre d'espèces animales et végétales (Atlas du parc national d'al Hoceima).

\section{- Biodiversité :}

Le Maroc est parmi les cinq premiers pays floristiquement les plus riches à l'échelle du Bassin Méditerranéen (Fennane, 2004) 
Tableau 1: Biodiversité floristique des pays du Bassin Méditerranéen (îles exclues) d'après Quezel \& Medail, 1994.

\begin{tabular}{|l|l|l|}
\hline Pays & $\begin{array}{l}\text { Surfaces en } \\
\text { région } \\
\text { méditerranéenne. }\end{array}$ & $\begin{array}{l}\text { Nombre d'espèces en } \\
\text { région méditerranéenne }\end{array}$ \\
\hline Maroc & $300000 \mathrm{~km}^{2}$ & 3800 \\
\hline Algérie & 300000 & 2700 \\
\hline Tunisie & 100000 & 1600 \\
\hline Lybie & 100000 & 1400 \\
\hline Egypte & 15000 & 1100 \\
\hline Jordanie & 10000 & 1800 \\
\hline Syrie & 50000 & 2600 \\
\hline Liban & 10000 & 2600 \\
\hline Turquie & 480000 & 5000 \\
\hline Grèce & 100000 & 4000 \\
\hline Italie & 200000 & 3850 \\
\hline France & 50000 & 3200 \\
\hline Espagne & 400000 & 5000 \\
\hline Portugal & 70000 & 2500 \\
\hline
\end{tabular}

Le PNAH héberge de nombreuses espèces d'intérêt pour la conservation en Méditerranée (Tableau 2), ces espèces se reproduisent pour la plupart et se maintiennent dans le site. De plus, elles sont comme des espèces communes dans le PNAH (UNEP-MAP RAC/SPA, 2009).

Ces espèces sont au nombre de 42 au total, elles sont représentées par 12 espèces d'algues (dix Fucophyta et deux Rhodophyta), 11 invertébrés (deux éponges, deux cnidaires, quatre mollusques, deux crustacés et un échinoderme), et 19 vertébrés (un poisson, cinq tétrapodes et 13 oiseaux). Parmi les espèces de vertébrés, le phoque moine de Méditerranée Monachus monachus, espèce emblématique du PNAH qu'il fréquentait régulièrement il y a quelques années, a été exclu de la liste car il semble que cette espèce est éteinte de la Méditerranée marocaine ( UNEP-MAP RAC/SPA, 2009).

Tableau 2 : Espèces d'intérêt pour la conservation en Méditerranée observées dans le PNAH (source : UNEP-MAP RAC/SPA, 2009)

\begin{tabular}{|l|l|l|l|l|l|l|l|l|}
\hline & LR & EU & CBa & CBe & UICN & CITES & $\begin{array}{l}\text { Abondance } \\
\text { rel. } \\
\text { (C) (NC) } \\
\text { (O) }\end{array}$ & $\begin{array}{l}\text { Statut } \\
\text { global } \\
\text { (r) (e) (m) }\end{array}$ \\
\hline MACROPHYTA & & & & & & & & \\
\hline Fucophyta & & & & & & & & \\
\hline Cystoseira amentacea & + & & II & I & + & & $(\mathrm{C})$ & $(\mathrm{m})$ \\
\hline Cystoseira elegans & + & & & & + & & $(\mathrm{C})$ & $(\mathrm{m})$ \\
\hline Cystoseira zosteroides & + & & II & I & + & & $(\mathrm{C})$ & $(\mathrm{m})$ \\
\hline Laminaria ochroleuca & + & & & $\mathrm{I}$ & + & & $(\mathrm{C})$ & $(\mathrm{m})$ \\
\hline
\end{tabular}




\begin{tabular}{|c|c|c|c|c|c|c|c|c|}
\hline Laminaria rodriguezii & + & & II & I & + & & ( C ) & $(\mathrm{m})$ \\
\hline Phyllariopsis brevipes & & & & & + & & ( C ) & (m) \\
\hline Phyllariopsis purpurascens & + & & & & + & & $(\mathrm{C})$ & $(\mathrm{r})(\mathrm{m})$ \\
\hline Saccorhiza polyschides & + & & & & + & & ( C ) & $(\mathrm{m})$ \\
\hline Sargassum vulgare & & & & & + & & ( C ) & $(\mathrm{m})$ \\
\hline Zonaria tournefortii & & & & & + & & ( C ) & $(\mathrm{m})$ \\
\hline \multicolumn{9}{|l|}{ Rhodophyta } \\
\hline Lithophyllum byssoides & + & & II & I & + & & ( C ) & $(\mathrm{m})$ \\
\hline Peyssonnelia squamaria & & & & & II & & ( C ) & $(\mathrm{m})$ \\
\hline \multicolumn{9}{|l|}{ INVERTEBRATA } \\
\hline \multicolumn{9}{|l|}{ Porifera } \\
\hline Axinella sp. & & & II & II & & & $(\mathrm{NC})$ & $(\mathrm{m})$ \\
\hline Ircinia sp. & & & II & II & & & ( C ) & $(\mathrm{m})$ \\
\hline \multicolumn{9}{|l|}{ Cnidaria } \\
\hline Astroides calycularis & & & II & II & & & $(\mathrm{C})$ & $(\mathrm{m})$ \\
\hline Corallium rubrum & & $\mathrm{V}$ & III & III & + & & $(\mathrm{O})$ & $(\mathrm{m})$ \\
\hline \multicolumn{9}{|l|}{ Mollusca } \\
\hline Charonia lampas & & & II & II & & & $(\mathrm{NC})$ & $(\mathrm{m})$ \\
\hline Cymbula nigra & & & II & II & & & $(\mathrm{NC})$ & $(\mathrm{m})$ \\
\hline Patella ferruginea & & IV & II & II & & & ( C ) & $(\mathrm{m})$ \\
\hline Lithophaga lithophaga & & IV & II & II & & II & $(\mathrm{NC})$ & $(\mathrm{m})$ \\
\hline \multicolumn{9}{|l|}{ Crustacea } \\
\hline Maja squinado & & & III & III & & & $(\mathrm{C})$ & - \\
\hline Scyllarides latus & & $\mathrm{V}$ & III & III & & & $(\mathrm{NC})$ & - \\
\hline \multicolumn{9}{|l|}{ Echinodermata } \\
\hline Paracentrotus lividus & & & III & III & & & (C) & - \\
\hline \multicolumn{9}{|l|}{ VERTEBRATA } \\
\hline \multicolumn{9}{|l|}{ Pisces } \\
\hline Epinephelus marginatus & & & III & III & & & $(\mathrm{NC})$ & - \\
\hline \multicolumn{9}{|l|}{ Tetrapoda } \\
\hline \multicolumn{9}{|l|}{ Reptilia } \\
\hline Caretta caretta & & II & II & II & + & I & $(\mathrm{O})$ & $(\mathrm{m})$ \\
\hline Dermochelys coriacea & & II & II & II & + & I & $(\mathrm{O})$ & $(\mathrm{m})$ \\
\hline \multicolumn{9}{|l|}{ Mammalia } \\
\hline Delphinus delphis & & II & II & II & + & II & $(\mathrm{NC})$ & $(\mathrm{m})$ \\
\hline Stenella coeruleoalba & & II & II & II & + & II & $(\mathrm{NC})$ & $(\mathrm{m})$ \\
\hline Tursiops truncatus & & II & II & II & + & II & $(\mathrm{NC})$ & $(\mathrm{m})$ \\
\hline
\end{tabular}




\begin{tabular}{|l|l|l|l|l|l|l|l|l|} 
Aves & & & & & & & & \\
\hline Aquila heliaca & & & & II & + & I & (O) & (m) \\
\hline Calonectris diomedea & & & II & II & + & & (O) & (m) \\
\hline Falco eleonorae & & & II & II & + & & (O) & (m) \\
\hline Hieraetus fasciatus & & & & II & + & & (O) & (m) \\
\hline Hydrobates pelagicus & & & II & II & + & & (O) & (m) \\
\hline Larus audouinii & & & II & II & + & & (NC) & (m) \\
\hline Milvus milvus & & & & II & + & & (NC) & (m) \\
\hline Pandion haliaetus & & & II & II & + & & (NC) & ( $)(\mathrm{m})$ \\
\hline Phalacrocorax aristotelis & & & II & II & & & (C) & (m) \\
\hline Phalacrocorax pygmaeus & & & II & II & & & (C) & (m) \\
\hline Sterna albifrons & & & II & II & + & & (O) & (m) \\
\hline Sterna bengalensis & & & II & II & + & & (O) & (m) \\
\hline Sterna sandvicensis & & & II & II & + & & (C) & (m) \\
\hline
\end{tabular}

Légende: (LR) Livre rouge de Méditerranée UNEP/IUCN/GIS (1990); (EU) Directive Habitat de l'Union Européenne (1992); (CBa) Plan d'Action pour la Méditerranée, Convention de Barcelone (1995); (CBe) Convention de Berne (1996); (UICN) Union Internationale pour la Conservation de la Nature; (CITES) Convention sur le Commerce des espèces de faune et flore sauvages menacées d'extinction; Abondance relative des espèces comme Commune (C), Non-Commune (NC) ou Occasionnelle (O); Son statut global des espèces comme Rare (r), Endémique (e) et/ou Menacé (m).

De plus, la faune halieutique est particulièrement riche et abondante, composée d'espèces méditerranéennes et atlantiques provenant du détroit de Gibraltar, qui représente une source d'alimentation de balbuzard pêcheur (Nibani H., 2010).

Récemment, la Direction des Eaux et Forêts, les gestionnaires, les organisations Non Gouvernementales (ONG), les chercheurs ont mis l'accent sur la conservation de certains espèces parmi eux on cite : le Thuya de Berbérie et le Balbuzard Pêcheur.

\section{Une grande biodiversité floristique... cas de thuya}

Le thuya de Berbérie est une essence qui est essentiellement cantonnée en région méditerranéenne méridionale occidentale, c'est au Maroc que cette espèce individualise les peuplements les plus étendus et les plus diversifiés, son aire de répartition s'étend en gros dans la partie orientale du pays, sur le Plateau central, et dans le secteur de l'arganier (cf. supra) (Benabid, 1985).

Dans le cadre de programme décennal 2015-2024, on collaboration avec la Direction Provinciale des Eaux et Forêts, des travaux de reboisement ont été réalisé dans le but de reconstituer les peuplements de Thuya et de restaurer les sols et leur défense contre l'érosion 


\section{Superficie en ha}

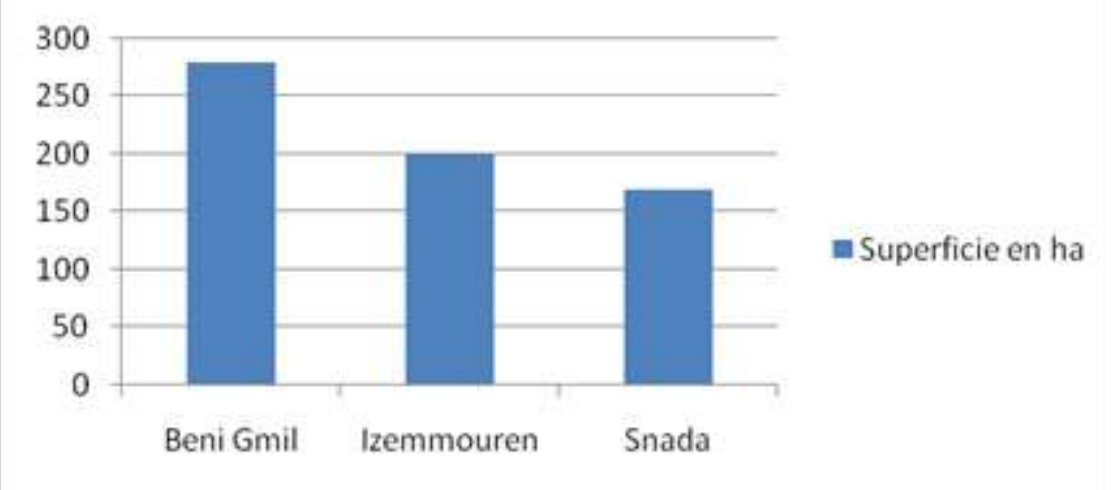

Graphe 2 : Travaux de reboisement de Thuya de berbérie au niveau du PNAH de l'année $2016 / 2017$

La tétraclinaie reste la formation forestière principale qui domine les peuplements forestiers au niveau du Parc National d'Al Hoceima sur 5014 ha

En effet, pour l'année 2016/2017 l'opération de reconstitution de la tétraclinaie c'est pour un total de 650 ha réparti entre Beni gmil et Izemmouren et Snada. Cette opération consiste à réhabilité ces peuplement et conserver les potentialités de l'écosystème.

Cette étude permet d'assurer à cette forêt un avenir dans lequel elle peut jouer ces rôles de production, protection et récréation, les objectifs assignés à cet aménagement sont : la préservation et la reconstitution des peuplements naturels, la réhabilitation de la forêt par la voie de reboisement, la production du bois de feu et de service, le développement des activités génératrices de revenus par une bonne mise en valeur des produits forestiers et de l'éco- tourisme, le développement des actions de partenariat et l'encouragement de l'adhésion des populations locales au processus de gestion durable des espaces naturels forestiers

Pour assurer le développement, la reconstitution et la sauvegarde des écosystèmes forestiers, les actions à entreprendre dans le cadre de la mise en œuvre du présent aménagement, s'intègrent dans la stratégie globale de développement et de conservation des ressources naturelles telle que définie par le Haut-Commissariat aux Eaux et Forêts et à la lutte contre la désertification.

\section{... et faunistique cas de balbuzard}

Le promoteur principal de la création du parc est de conserver le balbuzard, inscrit sur la liste rouge des espèces en danger de l'UICN (Union Internationale pour la Conservation de la Nature). C'est l'emblème du Parc National d'Al Hoceima, qui compte la seule population reproductrice au Maroc de ce rapace piscivore. Il niche dans les falaises les plus abruptes et les 
plus inaccessibles du Parc. Cependant, il est souvent victime de la pêche illégale et du dérangement (HCEFLCD, 2012). Le parc c'est parmi les plus endroits actif au monde de cette espèce.

Les falaises du parc national d'al Hoceima appartiennent au massif calcaire du Bokkoya qui se caractérise par une quarantaine de kilomètres abritant une importante diversité biologique qui a mis en valeur cet endroit citant Balbuzards (Pandion haliaetus), mouettes Audouins (Larus audouinii), le Faucon pérlerin (Falco peregrinus.) etc.

Le Haut-Commissariat aux Eaux et Forêts et à la Lutte Contre la Désertification du Maroc, les gestionnaires du Parc et les ONG ont lancé un programme de restauration des habitats du Balbuzard pêcheur.

Les suivis et les observations annuels ont été faits le long des falaises de Parc National d'Al Hoceima, de port d'Al Hoceima jusqu'au le port de Cala Iris.

Tableau 3 : Les effectifs des nids des Balbuzards pêcheur (Agir \& El Hadri, 2014)

\begin{tabular}{|l|l|l|l|}
\hline Année & $\begin{array}{l}\text { Nombre des } \\
\text { nids }\end{array}$ & $\begin{array}{l}\text { Nombre des } \\
\text { couples }\end{array}$ & $\begin{array}{l}\text { Nids } \\
\text { abandonné }\end{array}$ \\
\hline 1983 & 24 & 14 & 10 \\
\hline 1984 & 9 & 6 & 3 \\
\hline 1985 & 32 & 15 & 17 \\
\hline 1986 & 33 & 15 & 18 \\
\hline 1987 & 37 & 16 & 21 \\
\hline 1989 & 33 & 11 & 22 \\
\hline 1990 & 43 & 13 & 31 \\
\hline 2012 & 16 & 8 & 8 \\
\hline 2013 & 23 & 10 & 13 \\
\hline $\mathbf{2 0 1 4}$ & $\mathbf{3 1}$ & $\mathbf{1 0}$ & $\mathbf{2 1}$ \\
\hline
\end{tabular}

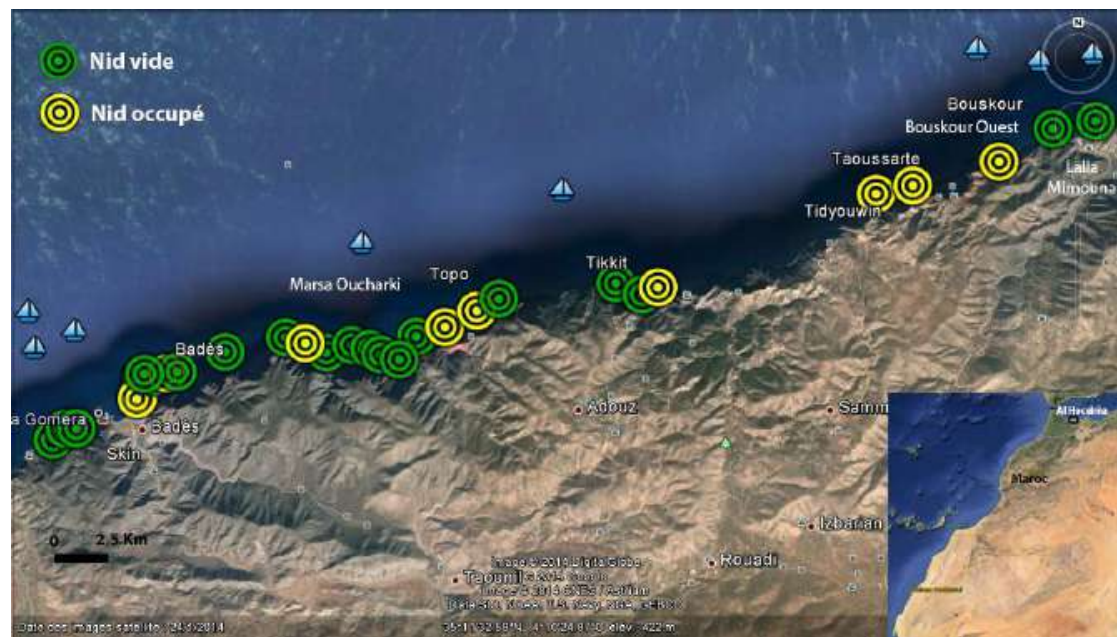

Figure 2 : La répartition des nids des Balbuzards pêcheur au PNAH (Source : El Hadri, 2014)

Deux types de facteurs pourraient expliquer pourquoi certaines structures de nidification ont disparu au cours des dernières décennies. 
Premièrement, les facteurs environnementaux tels que le vent et la pluie auraient pu détruire les nids inutilisés. Deuxièmement, les habitants des villages voisins explorent souvent les falaises côtières et utilisent la pêche à la dynamite et leurs passages répétés avec des explosions continues peuvent avoir accéléré le processus de démolition des nids. Ces deux facteurs pourraient être responsables de la destruction rapide des nids.

Ainsi que le stock du poisson est assez insuffisant et le taux de dérangement est élevé pour que tous les nids soient occupés, et comme la carte (figure 2) montre que les nids sont proches entre eux. On peut aussi expliquer cette diminution de la population du Balbuzard qu'au moment des vols nuptiaux les rapaces entrent en compétition entre eux pour réussir d'installer sur les nids avec les partenaires et on sait bien qu'un couple s'installe que sur un nid personnel.

\section{Destination écotouristique}

Le développement du tourisme balnéaire au Maroc, timide au cours des décennies précédentes et agressif depuis le contrat programme et le Plan Azur, intervient dans un contexte de littoralisation de l'économie et de l'espace (Berriane, 2007).

Le « tourisme durable » est un concept qui cherche à remédier aux problèmes environnementaux et sociaux engendrés par le tourisme (Erik, 2009). Ainsi il se présente comme un moyen permettant de valoriser les ressources des aires protégées, et engendrer un développement viable et soutenable (Chaoui, 2013).

Le tourisme participe aussi à la préservation de l'environnement, car la population locale s'encourage d'améliorer ses endroits pour attirer les touristes et préserver la beauté de ses entourages et le patrimoine local ainsi pour un développement économique, écologique et touristique durable. Le tourisme rural et de montagne et l'aménagement de la montagne à ces fins est une nouvelle orientation engagée en Europe après la seconde guerre mondiale (Mesplier \& Bloc-Duraffour, 1997)

Le parc national d'al Hoceima représente un agréable vue ainsi la ville est parmi les plus beaux arrière plan du Maroc ce qui est un point favorable pour attirer les touristes de profiter de la beauté du paysage, ainsi la richesse animale et végétale caractérisant ce parc rende cet espace comme un centre d'accueil pour les chercheurs biologiques écologiques, aussi les organismes non gouvernementaux (ONG) font des projets concernant la préservation de la biodiversité pour assurer un meilleur développement durable.

En fait, ces beaux panoramas ne manquent que des centres d'hébergement pour se détendre au milieu de ce patrimoine, le tableau ci-après (tableau 3) représentent en détail les gites et camping disponibles au niveau du parc national d'al Hoceima : 
Tableau 3 : représente les hébergements disponibles au niveau du PNAH (source : enquête 2019)

\begin{tabular}{|c|c|c|c|}
\hline Hébergements & Date de création & Capacité & Composition \\
\hline Gite de Taoussarte & Août 2005 & 8 & deux chambres \\
\hline Gîte de Taounil & Août 2008 & 8 & deux chambres \\
\hline Gite Rural Jnanate & Avril 2009 & 25 & cinq chambres \\
\hline Gîte d'Adouz & Juillet 2005 & 12 & deux chambres \\
\hline Camping Amis de Cala Iris & Juillet 2011 & 40 & $\begin{array}{c}\text { quatre chalets, } \\
\text { deux studios, } \\
\text { deux tentes caidale, } \\
\text { un cabanon Bambou, } \\
\text { un appartement, } \\
\text { éco lodge, } \\
\text { cinq mobiles homes }\end{array}$ \\
\hline
\end{tabular}

D'après le tableau (Tableau 3), on remarque que les gites qui s'installent au territoire du parc national ouvrent ses portes à partir de la création de l'aire protégé. On trouve la disponibilité de quatre gites (gite de Taoussarte, gite de Taounil, gite rural Jnanate et gite d'Adouz) et un camping accueillant les touristes ce qui mise en valeur cet endroit.

Les hébergements sont répartis le long du trajet du parc près des falaises littoral méditerranéen, ils ont une vue sur la mer caractérisant par une architecture traditionnelle marocaine, sont bien équipés en utilisant que des produits biodégradable dans le but de la préservation de l'écosystème local afin d'assurer une meilleure relaxation avec des jardins annexes offrant des fruits frais et bio et des vues panoramiques avec un mélange de terre et mer.

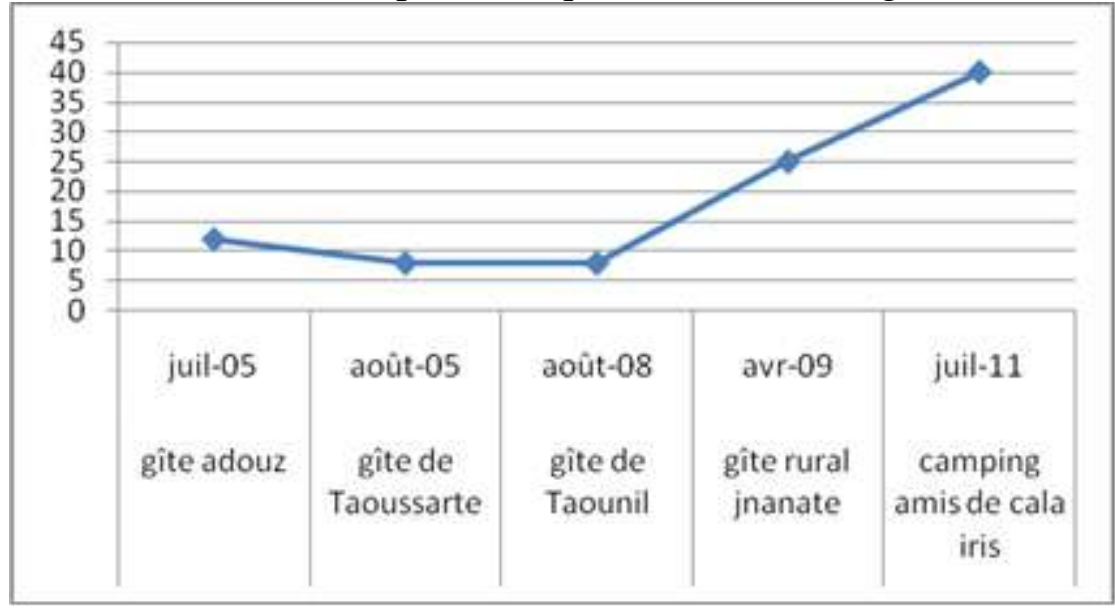

Graphe 3 : le développement la capacité des hébergements localisés au PNAH

Le graphe (Graphe 3) montre que le développement de la potentialité des chambres augmente de cinq personnes pour le gite Taoussarte en 2005 jusqu'à quarante personnes pour le Camping amis de Cala Iris en 2011, et 
récemment il y a des travaux de construction d'un nouveau hébergement dans la parcelle de Cala Iris. De plus certains habitants locaux transforme ses habitats comme un centre d'accueil, cela signifie que le nombre des visiteurs augmente par rapport qu'avant la création du parc national d'al Hoceima qui a été un espace négligeable et inconnu par contre qu'aujourd'hui qu'il a transformé par un espace protégé et divertissant et bon endroit pour pratiquer les loisirs et profiter de ses paysages et aussi c'est une bonne occasion de connaitre les monuments historique tel que le monument de Torres qui est un endroit peu visité pas les touristes externes, les coutumes et les traditions de la région afin de garantir un développement écotouristique utile et durable.

\section{Conclusion}

Même si l'existence des atouts et les potentialités biologiques et géologiques importantes, le PNAH connaît des menaces complexes, la pêche à la dynamite et avec des substances chimiques qui cause la surexploitation des ressources halieutiques, le mal utilisation des ressources naturelles par certaine population locale, le dérangement de la population des espèces le cas de balbuzard pêcheur par les pêcheurs artisanaux pratiquant leurs métiers près à des endroits de nidification, le surpâturage, etc.

Pour faire face à ces contraintes; des compagnes de sensibilisation destinées aux jeunes génération et aux exploitants de milieu pour savoir l'importance des ressources de ses entourages pour faire les impliquer à la gestion raisonnable de l'environnement ainsi pour conserver et améliorer la beauté du Parc National D'al Hoceima; d'améliorer et d'aménager les parcours propre aux visiteurs du parc aussi de faire diffuser ce lieu sur les médias afin de favoriser le secteur touristique qui va apporter un revenu intéressant aux propriétaires des hébergements afin de promouvoir « une gestion forestière écologiquement appropriée, socialement bénéfique et économiquement viable».

\section{References :}

1. Atlas du Parc National d'Al Hoceima, pp. 6-26.

2. Et-tobi M., Mhirit O. \& Benziane M., (2008). Changements climatiques, dégradations et dépérissements : Arguments et nouveaux outils pour une réforme de la sylviculture des cédraies au Maroc. 3ème Assises de la Recherche Forestière "L'écosystème cédraie : Un enjeu stratégique pour le développement régional » à Khénifra-Maroc, pp 2.

3. Ben Haj S., Bazairi H. \& Benhissoune S., (2009). UNEP-MAP RAC/SPA. Diagnostic de la biodiversité marine du Rif central (Méditerranée, Maroc) et orientations de gestion. Contrat CAR/ASP, $\mathrm{N}^{\circ}$ 47, 48 et 49: 100 pages, pp. 24-26. 
4. Benabid A., (1985). Les Écosystèmes Forestiers Pré Forestiers Et Presteppiques Du Maroc Diversité, Répartition Biogéographique Et Problèmes Posés Par Leur Aménagement. Forêt méditerranéenne, t. VII, $\mathrm{n}^{\circ} 1, \mathrm{pp} 4$.

5. Berriane M., (2007). Promouvoir un tourisme durable au Maroc Etude nationale du Maroc. Suivi de la stratégie méditerranéenne pour le développement durable, pp 17.

6. Chaoui MS., (2013). Le tourisme durable dans les aires protégées en Algérie : L'étude de cas du parc national de Taza, Université de Constantine, pp 1.

7. El Hadri S., (2014). Mémoire du stage fin d'études du Master .Contribution à la conservation de la vulnérable population du Balbuzard pêcheur du Parc National d'Al Hoceima, Maroc : Suivi, Régime alimentaire et Menaces. Tétouan. Maroc, pp. 44-45.

8. Erik E., (2009). Développement d'une stratégie de tourisme durable dans les aires protégées du Maroc. Tome 1 / ...-Berlin: SLE, pp 2.

9. Fennane M., (2004). Propositions De Zones Importantes Pour Les Plantes Au Maroc (ZIP Maroc). Atelier national "Zones Importantes de Plantes au Maroc", Rabat 11-12 /10/ 2004, pp 4.

10. Haut Commissariat aux Eaux et Forêts et à la Lutte Contre la Désertification, 2012. Faune Et Flore Du Parc Nationa D’Al Hoceima. Edition: UICN-Med - SEO/BirdLife, pp 27.

11. Haut Commissariat aux Eaux et Forêts et à la Lutte Contre la Désertification. Cadrage à 1'horizon 2020 du Plan Directeur des Aires Protégées au Maroc, pp 6.

12. Haut Commissariat aux Eaux et Forêts et à la Lutte Contre la Désertification. Guide des forêts urbaines et périurbaines. Édition 2010, pp 7.

13. Mesplier A. \& Bloc-Duraffour P., (1997). Le tourisme dans le monde. 5ème édition, Bréal, Rosny Cedex.

14. Najim L., (2001). Parc National D’al Hoceima-MAROC, pp 3.

15. Nibani H., (2010). Recherche et planification participative du secteur de la pêche artisanale à travers l'analyse systémique et prospective de durabilité dans la Zone Marine du Parc National d'Al Hoceima. Haut Commissariat aux Eaux et Forets et la lutte contre la désertification, Maroc.

16. Quezel P. \& Medail F., (1994). La région circum-méditerranéenne, centre mondial majeur de biodiversité végétale. Actes 6èmes Rencontres de l'A.R.P.E.: 152-161, Gap, France.

17. Royaume de Maroc. Ministère des eaux et forets. Brochure Parc national d'al Hoceima, pp 9. 
18. Tunesi et al., 2003. Plan De Gestion De La Composante Marine Du Parc National D'al Hoceima. Projet Régional pour le Développement d'Aires Protégées Marines et Côtières dans la Région Méditerranéenne (Projet MedMPA), pp. 25-99. 